\title{
INTELIGENCIA EMOCIONAL Y BULLYING EN ESCOLARES DE PRIMARIA
}

\author{
Cordelia Estévez Casellas \\ c.estevez@umh.es \\ Aída Carrillo \\ María Dolores Gómez-Medina \\ Centro Crimina \\ Universidad Miguel Hernández. España
}

Fecha de Recepción: 3 Marzo 2018

Fecha de Admisión: 10 Abril 2018

\section{RESUMEN}

Diferentes estudios señalan claras diferencias en los perfiles de inteligencia emocional asociados a los roles de víctima o agresor en el fenómeno del bullying (Casas, Ortega, Del Rey,2015). Estos estudios se centran habitualmente en población adolescente, pero existen menos estudios que analicen este fenómeno en menores de 11 años. El Estudio Cisneros (Piñuel, 2006) refleja que los índices de acoso escolar en primaria superan a los de secundaria, por esto consideramos necesario el análisis de las características emocionales de los menores de 11 años implicados en el bullying. El objetivo de este trabajo es analizar el perfil emocional de escolares de entre 8 y 11 años implicados en el bullying. Para ello se entrevistaron 291 niños de entre 8 y 11 años en los que se evaluaron las variables, Inteligencia emocional rasgo con el Inventario de Inteligencia Emocional BarOn ICE: NA (Bar-On, 2004), y Rol asociado al fenómeno de bullying con el Test Bull-S (Cerezo, 2002).

Los resultados confirma que la prevalencia de bullying en esta edad es alta (18\% agresores y $12 \%$ víctimas) siendo los chicos los que en la mayor parte de las ocasiones asumen el papel de agresor $(90 \%)$ y víctima $(67 \%)$.

Tras realizar un ANOVA de un factor se confirmó con un índice de significación $<0,05$ que los niños agresores puntuaban más bajo en el cociente de Inteligencia Emocional Global así como en las dimensiones Habilidades Interpersonales y Manejo del Estrés. A su vez las victimas presentaron puntuaciones inferiores en dichas dimensiones en comparación con los observadores pero más elevadas que las de los agresores.

Podemos concluir que el papel de la Inteligencia Emocional en los niños de primaria implicados en el fenómeno de bullying es un aspecto a tener en cuenta, a la hora de diseñar estrategias de prevención. 


\section{INTELIGENCIA EMOCIONAL Y BULLYING EN ESCOLARES DE PRIMARIA}

Palabras clave: inteligencia emocional; bullying; escolares; primaria

\section{ABSTRACT}

Emotional intelligence and bullying in elementary school children. Several studies detect clear differences in the profiles of emotional intelligence associated with the roles of victims or aggressors in the phenomenon of bullying (Casas, Ortega, Del Rey, 2015). These studies usually focus on the adolescent population, but there are fewer studies that analyze this phenomenon in children under 11 years of age. The Cisneros Studio (Piñuel, 2006) shows that rates of bullying in elementary schools outnumber high school, therefore it is necessary to analyze the emotional characteristics of children under 11 years involved in bullying.

The objective is to analyze the emotional profile school children between 8 and 11 years involved in bullying.

Methods: 291 children between 8 and 11 years of age in which the variables were evaluated trait emotional intelligence Emotional Intelligence Inventory BarOn were interviewed ICE: NA (Baron, 2004), and role associated with the phenomenon of bullying Test Bull-S. (Cherry, 2002)

It is confirmed that the prevalence of bullying at this age is high (18\% aggressors and victims $12 \%$ ) being boys who mainly assume the role of aggressor (90\%) and victim (67\%). One-way ANOVA analysis confirmed, with an index of significance $<0.05$, that aggressors scored lower on the Global Intelligence quotient as well as the dimensions interpersonal skills and stress management. In turn, the victims presented lower scores in these dimensions compared to the observers but higher than those of the aggressors.

We can conclude that the role of emotional intelligence in elementary school children involved in the phenomenon of bullying is an aspect to be taken into account, when designing prevention strategies

Keywords: emotional intelligence; bullying; elementary school; children

\section{INTRODUCCIÓN}

Los casos de acoso escolar conocidos se han incrementado en los últimos años, este hecho unido a los resultados de investigación que señalan las trágicas consecuencias que para la salud mental del menor tiene ser víctima de esta persecución sistemática (Springer, Cuevas, Ortiz, Keis y Wilkinson, 2015), hace que el interés científico y profesional por encontrar estrategias de prevención sea cada vez mayor

En un informe elaborado, en 2007, por el Gobierno Vasco sobre el maltrato entre iguales en los centros educativos, se destaca que la mejor prevención es aquella basada en las relaciones de la comunidad educativa, es decir, en el respeto, en la promoción de las conductas prosociales y en el uso del diálogo para el abordaje de conflictos. De esta manera, trabajar la empatía, la asertividad, aumentar las habilidades sociales, así como la educación emocional, además de educar en la no violencia y en la tolerancia a la diversidad cultural, son consideradas estrategias muy eficaces a la hora de prevenir el acoso escolar (Leganés, 2013; Save the Children, 2016).

Siguiendo en esta línea, el Centro de Salud Mental del Reino unido sostiene que las mediaciones escolares llevadas a cabo para reducir la problemática del bullying y las técnicas psicológicas aplicadas en los centros educativos, mejoran notablemente la repercusión de dicha problemática (Khan, Parsonage, Stubs, 2015). Es por ello que la Organización Save de Children resalta la importancia de incorporar la figura de un profesional, como puede ser la del psicólogo, con formación específica para llevar a cabo estrategias de prevención basadas en el aprendizaje emocional

Un estudio realizado por Cerezo (2008) demuestra que en las clases donde existen casos de acoso escolar, el índice de cohesión es bajo, por lo que no reina una clara pertenencia al grupo 0 
ésta es débil. En esta línea Fergus y Zimmerman (2005) señalan que el agresor tiene escasos niveles de habilidades sociales, para poder comunicar y negociar sus deseos, autocontrol y empatía. Además, éste también se caracteriza por ser una persona que necesita imponer sus deseos y justifica sus acciones asignando la causa de éstas a la necesidad de lograr sus objetivos (Avilés, 2006).

En referencia a las características emocionales de la víctima, un estudio realizado por Garaigordobil y Oñederra (2010) pone de manifiesto que las víctimas de acoso escolar presentan baja autoestima y bajo autoconcepto. Por otra parte, Cerezo (2001) señala que las víctimas suelen tener dificultades de comunicación, se muestran tímidas con poca asertividad y presentan inseguridad. Existen varios estudios que describen el hecho de que tanto la regulación emocional como la expresión de emociones (dimensiones ambas de la inteligencia emocional) puedan ser posibles predictores de victimización (Garner y Lemerise, 2007; Kelly, Schwart, Gorman y Nakamoto, 2008; Lemerise, Gregory y Fredstrom, 2005)

Por otra parte, Zavala y López (2012), hallaron que tanto las conductas prosociales como las altruistas en niños estaban directamente asociadas con altos niveles de inteligencia emocional. En esta misma línea, Fernández-Berrocal y Ruiz-Aranda (2008), señalan que la ausencia de habilidades de inteligencia emocional afecta a los estudiantes en todos los ámbitos de su vida diaria incluido el escolar, determinando los patrones relacionales que establecen los escolares en estos entornos.

Estos comportamientos disfuncionales prolongados y consistentes son un gran problema, ya que en uno de los indicadores de ajuste emocional en la infancia es la habilidad para relacionarse satisfactoriamente con sus iguales, sobre todo en el ámbito escolar, donde encuentran la segunda red principal de socialización. (Mateu-Martínez, Piqueras, Rivera-Riquelme, Espada et al, 2017; Springer, Cuevas, Ortiz, Keis y Wilkinson, 2015).

Existen indicios de que el rechazo social se relaciona con la fobia social, la disforia y la baja autoestima, es decir con problemas emocionales, y la aceptación social con habilidades sociales, es decir con la inteligencia emocional. Además también se ha indicado que los problemas conductuales de niños/as y adolescentes se debe a la falta de autorregulación emocional y la carencia de habilidades para expresar las emociones (Mateu-Martínez, Piqueras, Rivera-Riquelme; Melchor, 2017).

De hecho, existen estudios que señalan un perfil tanto de la víctima como del agresor. En el caso de la víctima destacan: menos conductas prosociales, falta de atención, conductas inmaduras, ansiedad, evitación, baja autoestima, autoconcepto negativo, inseguridad, depresión, insatisfacción con las relaciones de los compañeros y profesores, dificultad de aprendizaje, bajo rendimiento académico, abandono escolar, mayor sensibilidad al rechazo y mayor riesgo de problemas psiquiátricos en la adultez (Albores-Gallo et al., 2011; Cerezo, 2001; Garaigordobil y Oñederra, 2010; Plazas, Morón Cotes, Santiago, Sarmiento, et al., 2010; Estévez, Martínez y Jiménez, 2009). Por otra parte señalan que en el agresor destaca: bajos niveles de habilidades sociales en la comunicación y negociación de sus deseos, de autocontrol y de empatía, conductas de imposición, justificación de sus acciones por la necesidad de lograr sus objetivos y dificultad para comprender las emociones y sus causas (Avilés, 2006; Inglés, Torregrosa, García-Fernández, Martínez-Monteagudo, et al., 2014; Fergus y Zimmerman, 2005)

Además es importante añadir que existen varios estudios que aclaran que tanto la regulación emocional como la expresión de emociones pueden ser predictores de victimización del Bullying (Garner y Lemerise, 2007; Kelly, Schwart, Gorman y Nakamoto, 2008).

Por todo esto, en necesario destacar la importancia de la inteligencia emocional según la describió Ruben Bar On, es decir de las habilidades para reconocer, entender y utilizar las emociones, de relacionarse con otras personas, adaptarse a los cambios, resolver problemas de naturaleza personal e interpersonal, y de afrontar eficientemente las demandas y desafíos de la vida diaria (Bar- 


\section{INTELIGENCIA EMOCIONAL Y BULLYING EN ESCOLARES DE PRIMARIA}

On, 2006) en el desarrollo de los/as más pequeños/as. Así mismo, existen evidencia de que los alumnos que alcancen un nivel elevado en el dominio de estas competencias serán capaces de establecer y mantener más lazos afectivos con los demás durante la etapa escolar, mientras que aqueIlos que no consigan desarrollarlas presentarán más dificultades de adaptación, posibilidad de sufrir rechazo social y se verán afectados todos los ámbitos de su vida diaria como pueden ser: la familia, la escuela y el entorno social (Inglés, Torregrosa, García-Fernández, Martínez-Monteagudo, et al., 2014; Fernández-Berrocal y Ruiz, 2008; Mestre, Samper y Frías, 2004).

Por ello, pretendemos describir en este trabajo las competencias de inteligencia emocional de los menores implicados en el bullying y analizar si existen diferencias en función del rol adoptado.

\section{MÉTODO}

\section{Participantes}

La muestra estuvo conformada por 291 alumnos del Colegio de Educación Infantil y Primaria Víctor Pradera de la localidad de Elche, situada en la provincia de Alicante siendo el $23 \%$ alumnos de $3^{0}$ de primaria ( 8 años ), el $26,1 \%$ de $4^{0}$ de primaria ( 9 años ), el $26,1 \%$ de $5^{\circ}$ de primaria ( 10 años) y el $24,7 \%$ correspondiente a alumnos cursando 60 de primaria ( 11 años). En referencia al género un $55,7 \%$ son niños y el $44,3 \%$ restante pertenece a las niñas (Ver 1 y 2 )

Tabla 1.

Distribución de alumnos/as por género

\begin{tabular}{ccc}
\hline & \multicolumn{2}{c}{ Frecuencia } \\
\hline & $\mathrm{n}$ & $\%$ \\
$\begin{array}{c}\text { Masc } \\
\text { ulino }\end{array}$ & 162 & 55,7 \\
Feme \\
nino \\
Total & 129 & 44,3 \\
& 291 & 100 \\
\hline
\end{tabular}

Tabla 2.

Distribución de alumnos/as por curso.

\begin{tabular}{|c|c|c|}
\hline & \multicolumn{2}{|c|}{ Frecuencia } \\
\hline & $\mathrm{n}$ & $\%$ \\
\hline 3\%A & 25 & 8,6 \\
\hline 3ㅇ & 42 & $4^{14,}$ \\
\hline 4\%A & 25 & 8,6 \\
\hline 4으 B & 51 & $5^{17,}$ \\
\hline
\end{tabular}




\begin{tabular}{ccc} 
50A & 50 & $2^{17,}$ \\
5B & 26 & 8,9 \\
60A & 26 & 8,9 \\
60B & 46 & $8^{15}$ \\
& \multicolumn{2}{c}{100} \\
Total & 291 &, 0 \\
\hline
\end{tabular}

\section{Instrumentos}

La información sociodemográfica fue recogida a través de un cuestionario ad hoc, los datos obtenidos proporcionan información sobre el género, la edad, el curso y el estado civil de los padres. Para evaluar las variables de estudio se emplearon los siguientes instrumentos que reúnen las garantías psicométricas de fiabilidad y validez.

Para la evaluación de la Inteligencia Emocional se utilizó el BarOn ICE (2004): NA Abreviado (Bar-On, R.) que contiene 30 items distribuidos en 6 escalas: Las cuales se distribuyen en

Escala Intrapersonal: Hace referencia a la comprensión de los estados emocionales propios, asertividad y habilidad para visualizarse a sí mismo de manera positiva.

Escala Interpersonal: Referida a habilidades como la empatía y la responsabilidad social, capacidad de comprender los sentimientos ajenos.

Escala de Adaptabilidad: Evalúa la habilidad para resolver los problemas.

Escala de Manejo del estrés: Indica grado de tolerancia al estrés y control de impulsos.

Escala de Impresión positiva: Evalúa la percepción excesivamente favorable de sí mismo.

Finalmente tiene una escala que ofrece un Coeficiente Emocional total.

Este instrumento va dirigido a población de entre 7 y 18 años. El modo de aplicación puede ser individual o colectiva y utiliza una escala de tipo Likert de 4 puntos en la cual los evaluados responden a cada ítem según las siguientes opciones de respuestas: "muy rara vez", "rara vez", "a menudo" y "muy a menudo". Las puntaciones altas del inventario indican niveles elevados de inteligencia emocional y social. El coeficiente alfa de Cronbach para esta muestra fue de 081.

Para la evaluación del papel adoptado por el menor dentro del aula, en referencia al fenómeno del bullying, se utilizó un instrumento de evaluación sociométrica de la violencia entre escolares, cuya autora es Fuensanta Cerezo Ramírez.

Este cuestionario, Bull-S (Cerezo, 2000) se presenta en dos modalidades, forma A, para alumnos, y la forma P, para profesores. En este caso se utilizó la forma A para alumnos. El cálculo de la consistencia interna del instrumento se realizó mediante el Alfa de Cronbach, el rango de valores Alfa osciló entre .69 y .75. El valor de este indicador se incrementó cuando se separaron los ítems relacionados con los agresores de aquellos relacionados con las víctimas, siendo en este acaso el coeficiente alfa para las variables de agresión .82 y para las de victimización .83. Estos valores indican la constancia o estabilidad del instrumento y representan una fiabilidad de moderada a alta.

Así como también el Alfa de Cronbach con todos los ítems del cuestionario, incluyendo las variables sociométricas, fue de .68 . 


\section{INTELIGENCIA EMOCIONAL Y BULLYING EN ESCOLARES DE PRIMARIA}

\section{Procedimiento}

Una vez obtenidos los consentimientos informados de padres y tutores para la participación de los menores en este estudio, se procedió a la recopilación de datos mediante los cuestionarios descritos. Esta recopilación se llevó a cabo en varios días con la ayuda de psicólogos especializados en este campo.

Tras la recogida de los datos en los cursos establecidos, se procesaron mediante un programa estadístico y un programa de corrección especializado para la variable de bullying. El programa estadístico es el SPSS donde en primer lugar se realizaron los análisis descriptivos tanto de los alumnos como de las variables de Inteligencia Emocional como del Bull-S. Además, se obtuvieron resultados de las correlaciones entre dichas variables y las propiedades psicométricas de ambos instrumentos de evaluación.

Los análisis cuantitativos de los datos se realizaron mediante el programa informático SPSS empleando un nivel de significación de 0.05 .

\section{RESULTADOS}

Respecto al nivel de Inteligencia Emocional (IE), en la tabla 3 se detallan las puntuaciones obtenidas en el cuestionario BarOn ICE: NA abreviado. Se observan altos niveles de IE en cada una de sus dimensiones, así como en el Coeficiente Emocional Total. Aquellas variables donde se obtienen resultados más elevados son las habilidades Interpersonales, el Manejo del estrés y la Adaptabilidad.

Tabla 3.

Análisis descriptivo del Inventario de Inteligencia Emocional BarOn ICE.

\begin{tabular}{lcc}
\hline Dimensiones & Media & Desv. típ. \\
\hline Intrapersonal & 97,94 & 15,772 \\
Interpersonal & 110,94 & 33,997 \\
Adaptabilidad & 110,82 & 20,894 \\
Manejo del Estrés & 109,98 & 25,798 \\
& & \multicolumn{2}{c}{15,} \\
\hline
\end{tabular}

Con respecto al cuestionario Bull-S los resultados nos muestran que la variable con la medía más elevada $(x=2.70)$ es "Fuerte" con una desviación típica de 4.878. Por otra parte, la variable con la media más baja (1.75) es "Manía" con una desviación típica de 3.157. 
Tabla 4.

Análisis descriptivos del Test Bull-S.

\begin{tabular}{lcc}
\hline Dimensiones & Media & Desv. típ. \\
\hline Fuerte & 2,70 & 4,878 \\
Cobarde & 1,99 & 3,406 \\
Agresivo & 1,80 & 3,736 \\
Victima & 2,10 & 3,323 \\
Provoca & 1,89 & 3,742 \\
Manía & 1,75 & 3,157 \\
\hline
\end{tabular}

En la tabla 5 se muestran las diferencias de género con respecto al cuestionario Bull-S donde podemos observar que en el género masculino la variable donde más media se obtiene coincide con la de la muestra general, la cual es "Fuerte" ( $x=4.48)$ con una desviación típica de 5.880. En cambio en el femenino la variable con la media más elevada es "Víctima" (1.74) con una desviación típica de 3.442 Haciendo referencia a las variables con media más baja, en el género masculino sería "Manía" ( $x=1.99$ y ds=3.248) y en el femenino sería "Fuerte" ( $x=.47$ y ds=1.173).

Tabla 5.

Análisis descriptivos del Test Bull-S según género.

\begin{tabular}{cccc}
\hline Género & Dimensiones & Media & Desv. típ. \\
\hline & Fuerte & 4,48 & 5,880 \\
Masculino & Cobarde & 2,42 & 3,873 \\
& Agresivo & 2,62 & 4,519 \\
& Victima & 2,39 & 3,208 \\
& Provoca & 2,75 & 4,494 \\
& Manía & 1,99 & 3,248 \\
& Fuerte &, 47 & 1,173 \\
& Cobarde & 1,44 & 2,622 \\
& Agresivo &, 76 & 1,995 \\
& Victima & 1,74 & 3,442 \\
& Provoca &, 81 & 2,049 \\
& Manía & 1,46 & 3,026 \\
\hline & & &
\end{tabular}




\section{INTELIGENCIA EMOCIONAL Y BULLYING EN ESCOLARES DE PRIMARIA}

Según los datos recogidos por el cuestionario Bull-S hemos el porcentaje de niños identificados como agresores es 52 , (18\% de la muestra total) siendo la mayor parte de ellos (90\%) de género masculino. En referencia a las víctimas, en este estudio se identificaron de $36(12 \%)$ de los cuales un $33 \%$ fueron chicas. El resto se clasificaron en la categoría de observadores o no implicados (Tabla 6 y 7 ).

Tabla 6.

Análisis de frecuencia según categoría Bull-S.

\begin{tabular}{lcc}
\hline & \multicolumn{2}{c}{ Frecuencia } \\
\hline & $\mathbf{n}$ & $\%$ \\
\cline { 2 - 3 } Espectador & 203 & 70 \\
Victimización & 36 & 12 \\
Agresión & 52 & 18 \\
Total & 291 & 100 \\
\hline
\end{tabular}

Tabla 7.

Análisis de frecuencia según categoría Bull-S y género.

\begin{tabular}{llcc}
\hline & Género & \multicolumn{2}{c}{ Frecuencia } \\
\hline & & $\mathrm{n}$ & $\%$ \\
\cline { 3 - 4 } Espectador & Masculino & 91 & 45 \\
& Femenino & 112 & 55 \\
Victimización & Masculino & 24 & 67 \\
& Femenino & 12 & 33 \\
Agresión & Masculino & 47 & 90 \\
& & 5 & 10 \\
Total & Femenino & & 100 \\
\hline
\end{tabular}

Finalmente, se procedió analizar el nivel de inteligencia emocional total y dimensional en tiene cada una de las 3 categorías de alumnos extraídas del Bull-S (Alumnos no implicados, alumnos agresores y alumnos victimizados). En dicha tabla podemos observar que las medias de las variables "Interpersonal", "Manejo del Estrés", así como en la "Puntuación total", son más elevadas en la categoría espectadores que en la de victimización y ésta, a su vez, más elevada que en la de agresión. Siendo significativas con un nivel de $p<0,05$ (véase Tabla ). 
Tabla 1. Diferencias Inteligencia Emocional según categorías Bull-S.

\begin{tabular}{lll} 
Dimensiones & & ig. \\
\hline Intrapersonal &, 246 & 289 \\
Interpersonal &, 852 & $022^{*}$ \\
Adaptabilidad &, 531 & 081 \\
Manejo del Estrés & 0,977 & $000^{*}$ \\
Puntuación total & & \\
\hline
\end{tabular}

Tabla 9. ANOVA de Inteligencia Emocional y categorías Bull-S.

\begin{tabular}{|c|c|c|c|c|c|c|}
\hline Categc & ría Bull-S & Intrapersonal IE & Interpersonal IE & Adaptabilidad IE & Manejo del Estrés IE & Puntuación total IE \\
\hline \multirow[t]{3}{*}{ No implicado } & $\mathrm{N}$ & 203 & 203 & 203 & 203 & 203 \\
\hline & Media & 13,98 & 20,39 & 17,70 & 18,71 & 86,94 \\
\hline & Desv. Típ. & 3,322 & 2,787 & 3,225 & 3,434 & 8,962 \\
\hline \multirow[t]{3}{*}{ Victimización } & $\mathrm{N}$ & 36 & 36 & 36 & 36 & 36 \\
\hline & Media & 14,00 & 20,17 & 17,58 & 17,58 & 85,75 \\
\hline & Desv. Típ. & 3,780 & 3,291 & 3,901 & 3,698 & 11,768 \\
\hline \multirow[t]{3}{*}{ Agresión } & $\mathrm{N}$ & 52 & 52 & 52 & 52 & 52 \\
\hline & Media & 13,17 & 19,08 & 16,52 & 16,21 & 79,71 \\
\hline & Desv. Típ. & 3,066 & 3,808 & 3,638 & 3,707 & 10,610 \\
\hline
\end{tabular}

\section{DISCUSIÓN Y CONCLUSIONES}

En primer lugar es interesante como parte de la discusión de los resultados, señalar que la distribución víctima vs agresor en el fenómeno de bullying por géneros se mantiene tal y como señala la bibliografía (Andreu et al. 2012). Es llamativo que el porcentaje de chicos, tanto en víctima como en agresor, sea más elevado que el de chicas.

En cuanto a la edad los datos obtenidos en nuestro estudio al igual que los obtenidos por Cerezo en 2008 sugieren que la frecuencia de las agresiones es, claramente, mayor en 9 años, a pesar de que el fenómeno de bullying suela relacionarse habitualmente con la adolescencia. Este cambio de tendencia ya evidenciado por otros autores no solo es preocupante, si no que nos lleva 
a reflexiones más profundas acerca de los entornos educativos y el tipo de información o entretenimiento a los que están expuestos los más pequeños

Si centramos la atención en el aspecto, más relevante de este estudio, es decir sobre la relación entre inteligencia emocional y acoso escolar, es necesario resaltar que, existen diferencias significativas en esta variable según el rol adoptado en este fenómeno, es decir ser agresor, víctima o no implicarse. En primer lugar, encontramos según lo esperado que tanto los agresores como las victimas presentan cocientes de Inteligencia emocional inferiores a los de sus compañeros no implicados en el fenómeno del bullying, sabíamos que la ausencia o déficits en destrezas como la empatía o la autorregulación se presentaba como un factor de riesgo para implicarse activamente en este tipo de conductas (Garaigordobil y Oñederra ;2010), ahora sabemos que el hecho de disponer desde la infancia de estrategias emocionales que lleven a la identificación , regulación y /o reparación emocional puede aportar a los niño estrategias para evitar victimizarse 0 acosar a los compañeros.

Dentro de este perfil emocional y desde el modelo de Rubén Bar-On, encontramos que concretamente las habilidades relacionadas con las dimensiones "Relaciones Interpersonales" y "Manejo del estrés" las que también presentan diferencias significativas en los tres grupos estudiados. La dimensión "Interpersonal" de Bar-On, como ya hemos descrito anteriormente hace referencia a habilidades como la empatía y la responsabilidad social. Podemos comprobar que tanto los agresores como los que son víctimas tienen menos habilidades en estos aspectos que los niños no implicados, y que son los agresores que en general, y ajustándose a lo esperado, más bajo puntúan. Este aspecto es de especial importancia ya que no solo van en consonancia con los obtenidos por otros autores como Avilés y Monjas (2005) y Diez-Aguado (2006), si no, que nos dan un perfil de las habilidades emocionales que necesitan los menores para mejorar la convivencia en las aulas y señalan un camino para diseñar programas preventivos basados en la educación emocional de este tipo de habilidades interpersonales.

Por último, es interesante reflexionar sobre los resultados obtenidos en relación a las diferencias entre agresores, victimas y los menores no implicados en lo referente a la dimensión del manejo de estrés, para Bar On, esta dimensión hace referencia al grado de tolerancia al estrés del menor y a su habilidad para controlar los impulsos, aspectos fundamentales estos, a la hora de establecer relaciones sociales en la infancia. Referente a esto, son de nuevo los escolares que acosan y persiguen a sus compañeros los que toleran peor las frustraciones y tienen menos estrategias útiles para regular esta frustración, recurriendo probablemente a la agresión como vía de desahogo

Con todo lo expuesto anteriormente consideramos necesario resaltar el papel de la educación emocional en la infancia, no solo como parte de un abordaje global del proceso educativo si no como estrategia preventiva del acoso escolar.

\section{REFERENCIAS BIBLIOGRÁFICAS}

Albores-Gallo, L., Sauceda-García, J., Ruiz-Velasco, S., y Roque-Santiago, E. (2011). El acoso escolar (bullying) y su asociación con trastornos psiquiátricos en una muestra de escolares en México. Salud Pública de México, 53(3), 220-227.

Alonso, J. y Román, J. M. (2005). Prácticas educativas familiares y autoestima. Psicothema, 17, 7682.

Avilés, J. (2006). Bullying: el maltrato entre iguales. Agresores, víctimas y testigos en la escuela. Salamanca: Amarú.

Bar-On, R. (2006). The Bar-On model of emotional-social intelligence (ESI). Psicothema, 18, 13-25. Bornstein, L. y Bornstein M. H. (2007). Parenting styles and child development. Encyclopedia on 
Early Childhoop Development, 1-4. Montreal, Quebec: Centre of Excellence for Early Childhood Development.

Cerezo, F. (2001). Variables de personalidad asociadas en la dinámica bullying (agresores versus víctimas) en niños y niñas de 10 a 15 años. Anales de psicología 17, 37-43.

Cerezo, F. (2008). Agresores y Víctimas del Bullying. Desigualdades de género en la violencia entre escolares. Revista de Informació Psicológica. (94), 49-59.

Departamento de Educación, U. e. (2007). Guía de actuación en los centros educativos ante el maltrato entre iguales. Servicio Central de Publicaciones del Gobierno Vasco.

Estévez, E., Martínez, B. y Jiménez, T.I. (2009). Las relaciones sociales en la escuela: El problema del rechazo escolar. Revista de los Psicólogos de la Educación, 15(1), 45-60.

Fernández-Berrocal, P. y Ruiz-Aranda, D. (2008). Emotional intelligence in the school context. Electronic Journal of Research in Educational Psychology, 15.

Fergus, S., y Zimmerman, M. (2005). ADOLESCENT RESILIENCE: A Framework for Understanding Healthy Developmentin the Face of Risk. Annual Review of Public Health 26(1):; 399-419.

Furnham, A. y Cheng, H. (2004). Perceived parental behaviour and happiness. Social Psychiatry and Psychiatrics Epidemiology, 35, 463-470.

Garner, P. W., y Lemerise, E. A. (2007). The roles of behavioral adjustment and conceptions of peers and emotions in preschool children's peer victimization. Development and Psychopathology, 19, 57-71.

Garaigordobil, M. y Oñederra, J.A. (2010). Inteligencia emocional en las víctimas de acoso escolar y en los agresores. European Journal of Education and Psychology, 3(2), 243-256.

Gracia, E., Lila, M. y Musitu, G. (2005). Rechazo parental y ajuste psicológico y social de los hijos. Salud Mental, 28, 73-81.

Goleman, D. (1996): Inteligencia emocional. Barcelona, España. Kairós.

Heider, D., Matschinger, H., Bernet, S., Alonso, J. y Angermeyer, M. (2006). Relationship between parental bonding and mood disorder in six European countries. Psychiatry Research, 143, 8998.

Hidalgo, V. y Palacios, J. (1999). Desarrollo de la personalidad entre los 2 y los 6 años. Desarrollo psicológico y educación. Psicología evolutiva, 1, 257-304. Madrid: Alianza Editorial.

Inglés, C., Torregrosa, M., García-Fernández, J., Martínez-Monteagudo, M., Estévez, E., y Delgado, B. (2014). Conducta agresiva e inteligencia emocional en la adolescencia. European Journal of Education and Psychology, 7 (1), 29-41

Jabeen, F., Anis-ul-Haque, M. y Riaz, M. N. (2013). Parenting styles as predictors of emotion regulation among adolescents. Pakistan Journal of Psychological Research, 28(1), 85-105.

Khan, L., Parsonage, M., y Stubbs, J. (2015). Investing in children s mental health. A review of evidence on the costs and benefits of increased service provision. Centre for Mental Health Report.

Kelly, B. M., Schwartz, D., Gorman, A. H., y Nakamoto, J. (2008). Violent victimization in the community and children's subsequent peer rejection: the mediating role of emotion dysregulation. Journal of Abnormal Child Psychology, 36, 175-185.

Leganés Lavall, E. (2013). Una propuesta de intervención para prevenir el acoso escolar desde una perspectiva sociogrupal. Psychology, Society and Education, 5 (1), 21-40.

Lila, M. S. y Marchetti, B. (1995). Socialización familiar. Valores y autoconcepto. Informació Psicològica, 59, 11-17.

López-Soler, C.; Puerto, J.C.; López-Pina, J.A. y Prieto, M. (2009). Percepción de los estilos educativos parentales e inadaptación en menores pediátricos. Anales de psicología, 25, 70-77.

Maccoby, E. E. y Martin, J. A. (1983). Socialization in the context of the family: Parent-child inter- 
action. En E. M. Hetherington y P. H. MUSSEN (Eds.), Handbook of Child Psychology: Socialization, Personality, and Social Development, 4, 1-101. New York: Wiley.

Mateu-Martínez, O., Piqueras, J., Rivera-Riquelme, M., Espada, J., \& Orgilés, M. (2017). Aceptación/rechazo social infantil: relación con problemas emocionales e inteligencia emocional. Avances En Psicología, 22(2), 205-213. Consultado de http://revistas.unife.edu.pe/index.php/avancesenpsicologia/article/view/190

Melchor, D. (2017). Relación entre inteligencia emocional, conductas violentas y clima escolar en educación primaria (Tesis Doctoral). Universidad Miguel Hernández, España.

Méndez, I. y Cerezo, F. (2010) Test Bull-s: programa informático de evaluación de la agresividad entre escolares. En Arnaiz, P.; Hurtado, Mạ.D. y Soto, F.J. (Coords.) 25 Años de Integración Escolar en España: Tecnología e Inclusión en el ámbito educativo, laboral y comunitario. Murcia: Consejería de Educación, Formación y Empleo.

Mestre, V., Samper, P. y Frías, M. D. (2004). Personalidad y contexto familiar como factores predictores de la disposición prosocial y antisocial de los adolescentes. Revista Latinoamericana de Psicología, 36(3), 445-457.

Oliva, A., Parra, A. y Arranz, E. (2008). Estilos relacionales parentales y ajuste del adolescente. Infancia y Aprendizaje, 31, 93-106.

Plazas, E. A., Morón Cotes, M. L., Santiago, A., Sarmiento, H., Ariza López, S. E. y Patiño, C. D. (2010). Relaciones entre iguales, conducta prosocial y género desde la educación primaria hasta la universitaria en Colombia. Universitas Psychologica, 9(2), 357-369.

Ramírez-Lucas, A., Ferrando, M. y Sainz, A. (2015). ¿Influyen los estilos parentales y la inteligencia emocional de los padres en el desarrollo emocional de sus hijos escolarizados en $2^{\circ}$ ciclo de educación infantil? Acción Psicológica, 12 (1), 65-78.

Save the Children (2016). Yo a eso no juego: bullying y ciberbullying en la infancia. SAVE THE CHILDREN España.

Springer, A. E., Cuevas, M. C., Ortiz, Y. K., y Wilkinson, A. (2015). The association of bullying and school cohesion in Colombia secondaty school students. Global Health Promotion, 1757-9759, $1-12$.

Zavala, M. A., \& López, I. (2012). Adolescentes en situación de riesgo psicosocial: ¿Qué papel juega la Inteligencia Emocional? Behavioral Psychology / Psicología Conductual: Revista Internacional Clínica y De La Salud, 20(1), 59-75. 